\title{
Classification of Eyes Based on Fuzzy Logic
}

\author{
Mohamed Fakir, Faculty of Sciences and Technics, Beni Mellal, Morocco \\ Hatimi Hicham, Sultan Moulay Slimane University, Morocco \\ Mohamed Chabi, Faculty of Sciences and Technics, Beni Mellal, Morocco \\ Muhammad Sarfraz, Kuwait University, Kuwait \\ (iD https://orcid.org/0000-0003-3196-9132
}

\begin{abstract}
The systems of eye classification in an image are indispensable in several domains. To better find the class of membership of the eye in a minimal time, the classic methods of detection are inadequate. Fuzzy logic is considered to be an effective technique for solving an eye classification problem. This article proposes a fuzzy approach for eye classification. The tasks of classification are realized in two steps. In the first step, the characteristic points of the image are extracted in order to locate the eye. These characteristic points allow generating a representative model of the eye. In the second step, the detected eyes have to pass by a fuzzy controller containing several parts: Fuzzification, inference rules, and defuzzification. Finally, the system gives the degree of membership of the detected eyes to each class in the database.
\end{abstract}

\section{KEYWORDS}

Classification, Corner Detector, Defuzzification, Eye Detection, Eye Segmentation, Fuzzification, Fuzzy Controller, Inference System

\section{INTRODUCTION}

Classification and detection of the eyes in images is an important topic for new research (Christensen et al., 2017). Recently, researchers find solutions against Parkinson's disease based on retinal analysis (Dimililer et al., 2016). The classification of the human eye is a problem encountered in many fields, in medicine (Rigas \& Komogortsev, 2017), in the field of human-machine communication, in marketing and in the automobile industry (Minhad et al., 2017). In medicine for example, researchers are increasingly interested in the eye as a diagnostic tool for screening illnesses even before symptoms appear (Uchino et al., 2018). As result, the classification of the eyes is a very important operation, because the use of the eyes to authenticate a person is in great demand in the security systems. In this paper, we propose a new method for the classification of the eyes based on fuzzy logic. In fact, the mode of reasoning in fuzzy logic is more intuitive than classical logic, since we can have similarities between the eyes of several people. So, it's hard to decide who owns the eyes. The fuzzy logic makes it possible to find the eyes belonging's degree to each person which makes it possible to study all the possible results. The proposed method contains several steps that will allow us to minimize the general complexity of the algorithm. The results of this research can be used in biometrics to identify a person, or in the field of medicine to facilitate the task of physicians so as to make a decision. 
The most common classification methods can be divided into two main categories: supervised classification methods and unsupervised classification methods. The unsupervised classification is to let the computer automatically calculate the classes based on several features. Whereas in supervised classification, classes are created by user and the computer is let to assign the pixels to the classes. In this work, we will use a supervised method for classifying the eyes.

Classical logic is a set of mathematical notions that is relatively well known to the public; moreover, computers, calculators and most digital machines work depending on that principle. In classical logic, decisions are binary which are either true or false. Therefore, fuzzy logic is distinguished from classical logic. In fuzzy logic, a decision can be both true and false at the same time, with a certain degree of belonging to each of these two beliefs.

Currently, several studies in the classification field are based on fuzzy logic (Garcia-Arroyo \& Garcia-Zapirain, 2019). Moreover, there are methods that combine fuzzy logic and neural networks, which help to solve some complex problems (Ramirez et al., 2019). Other methods based on the algorithm of fuzzy c-means have been proposed (Zhang et al., 2018). The latter is based on the optimization of a quadratic classification criterion where each class is represented by its center of gravity.

This paper is organized as follows: section 2 deals with research method. Section 3 describes the fuzzy system detection proposed. In section 4, experimental results and discussion are given. The paper is ended by a conclusion in section 5 .

\section{RESEARCH METHOD}

The fuzzy system created in this paper allows giving the degree of membership of each eye located on an input image to the classes of the eyes recorded in a database (Figure 1):

Figure 1. Steps of detection used in the fuzzy controller

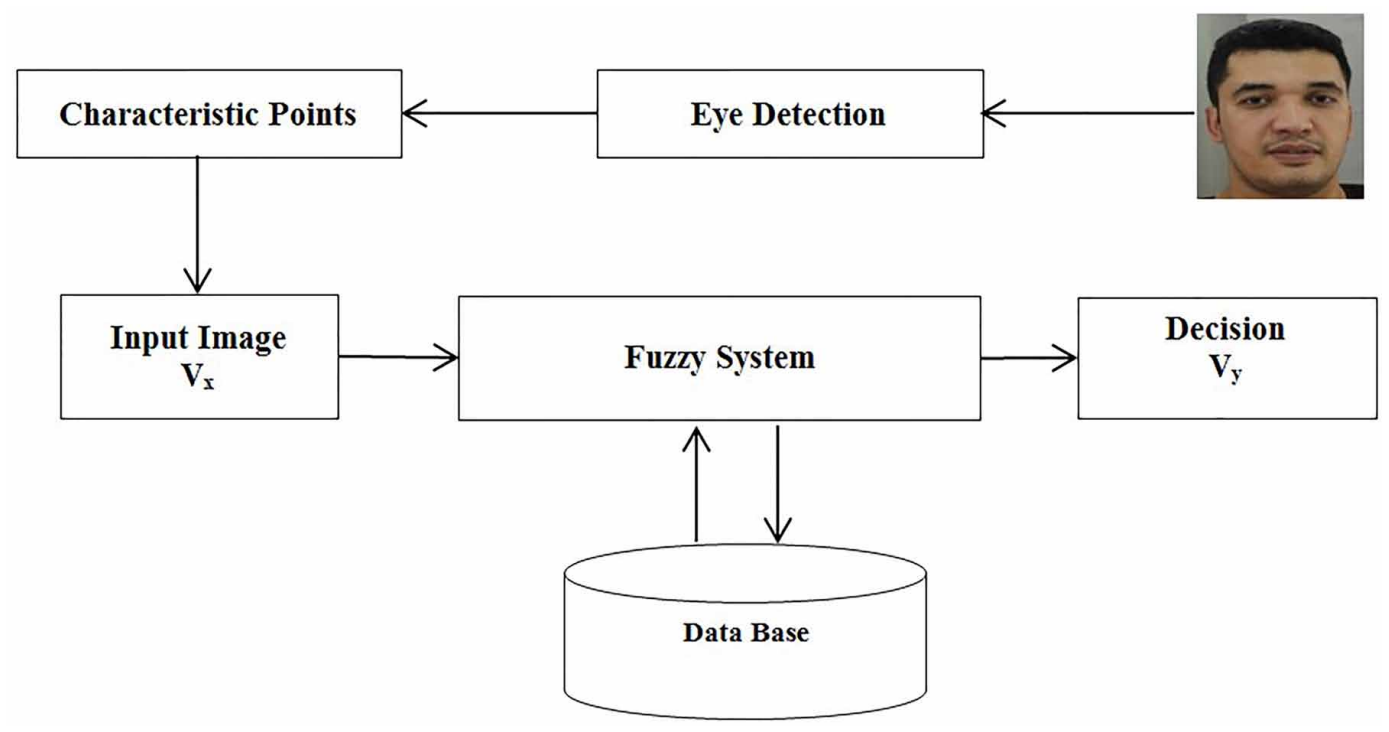

The fuzzy classification adopted in this paper consists of four steps (Figure 1). The first step locates the eyes from the face. Then in the second step, a point of interest detection algorithm (Harris 
\& Stephens, 1988) is applied in order to generate the characteristic vector of the input image. Next, a fuzzy classification system allows giving the belonging degree of the treated person to each class of the database. In the last step, only the classes with the highest degrees are remained in the final decision.

$\mathrm{V}_{\mathrm{x}}$ represents the characteristic vector of the eyes treated and $\mathrm{V}_{\mathrm{y}}$ represents the degree of belonging of the eyes to each class of the database.

The fuzzy system consists of three steps (Figure 2). The first step is fuzzification, the second step is the fuzzy inference system that contains a set of conditional rules, and the last step is defuzzification which gives the final result (Bloch, 2003).

Figure 2. Architecture of fuzzy system

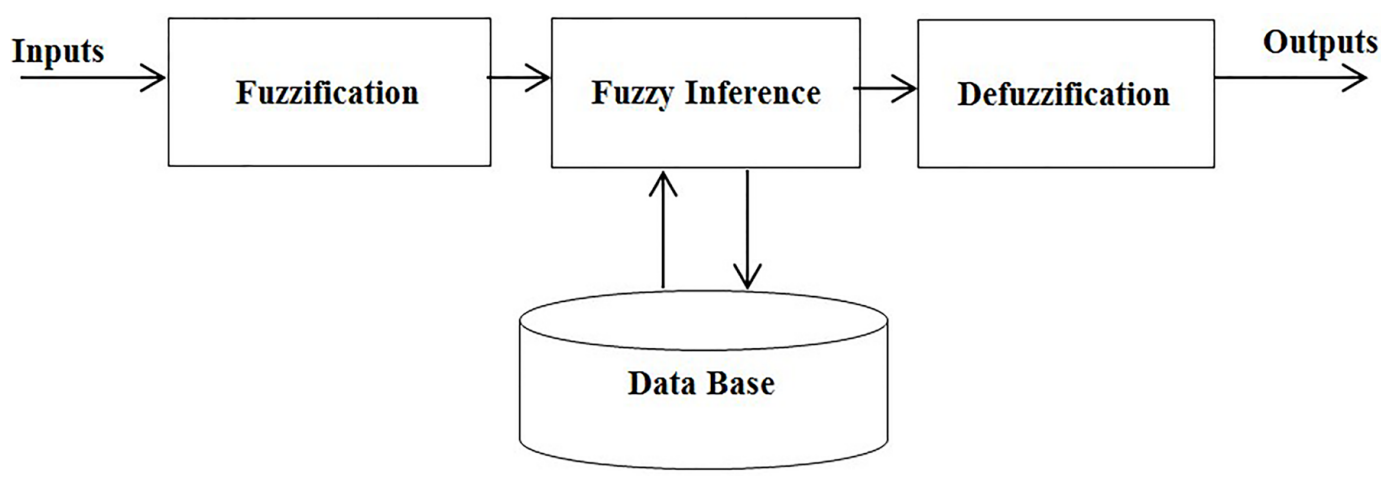

\section{Eye Detection}

Eye detection methods are divided into two categories; there are methods to determine if the eyes are present in the scene. The second type of method makes it possible to locate the eyes position. Each method takes a different approach, there are methods that use specific lighting (Cleveland, 1994). Other methods allow locating the eyes thanks to anatomical characteristics (Yuille et al., 1989; Chow \& Li, 1993; Song et al., 2010).

The method used in this paper to detect the eyes is based on extracting the encompassing rectangles from the eyes, nose and mouth (Viola \& Jones, 2001). It includes knowledge about the face, including the spatial distribution of different elements on the face. For example, the eyes are on the upper part of the face, the nose and mouth are aligned on the axis of symmetry of the face. In order to achieve good detections and despite the variation of scale, an energy map adapting to the scale of the face is proposed. Figure 3 shows the result of eye detection using the Viola and Jones method (Viola \& Jones, 2001):

This method (Cleveland, 1994) makes possible analyzing an image possible, without having to study each pixel constituting it. The notion of integral image makes it possible to define several rectangular zones within an image. The advantage of this technique lies in the fact that it offers the possibility of accessing the value of the other zones on the left and above the zone on which we are. These zones allow creating pseudo-Haar characteristics, which are in fact masks that allow to determine several patterns (Figure 4):

This method determines adjacent rectangles in order to calculate the sum of the pixel intensities of the image in these rectangular areas. The pseudo-Haar characteristics are the difference between black and white rectangles. These characteristics make it possible to detect patterns. For example, face recognition is made possible by the variation in the intensity of light between the eyes and the 

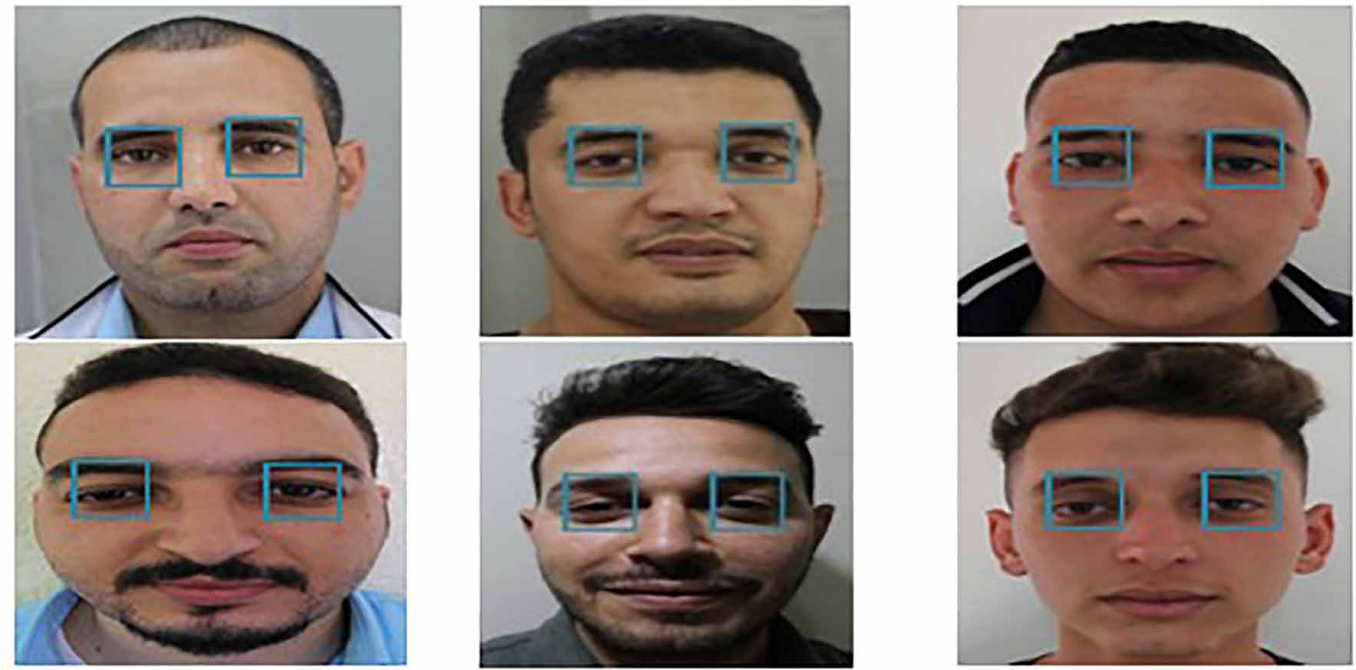

nose (characteristic number 2) and the variation in the intensity of light between the eyes and the cheekbones (characteristic number 3 in Figure 4).

\section{Preprocessing}

Once the data is acquired by a camera, it is often necessary to pre-process them in order to clean the signals and / or highlight interesting information that can be processed there. Indeed, the information can be easily disturbed electrically, by the muscular activity of the eyes or the face for example. To carry out this pretreatment, different space-time filters are used (Liu et al., 2014; Heuvelink et al., 2006). The most common are independent component analysis (ICA) and principal component analysis (PCA) (Hoya et al., 2003). In our method, a median filter was used to improve the quality of treatment (Lukac, 2003).

The role of the median filter in our approach is to improve the result of the extraction of interested points. It speeds up processing by minimizing detection errors. In this paper, the filter is applied to the eye area only before proceeding to the feature point extraction step.

\section{Classification}

In the classical approach, the goal of this step is to automatically assign a class to the previously extracted feature vector. This class represents the closest type of eyes to the treated ones. In this paper, we have created a fuzzy method for the classification of the eyes.

The object is to assign to a class all the eyes having a degree of membership to this class higher or equal to a given threshold, each eye now having the possibility to belong simultaneously to several classes.

The problem of blurring of the eyes can be formulated as follows:

Let $\mathrm{C}=\left\{\mathrm{C}_{1} ; \mathrm{C}_{2} ; \ldots ; \mathrm{C}_{\mathrm{m}}\right\}$ a set of $\mathrm{m}$ eye classes recorded in a data base. Let $\mathrm{V}_{\mathrm{xj}}$ a characteristic vector of the eye $\mathrm{j}$ of real $\mathrm{d}$ given by formula (1):

$V_{x j}=\left(e_{1} ; e_{2} ; \ldots ; e_{d}\right) \in \mathbb{R}^{d}$ 
Figure 4. Characteristics pseudo-Haar

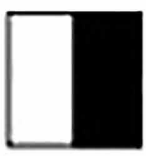

(1)

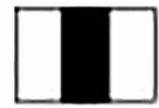

(5)

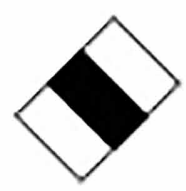

(9)

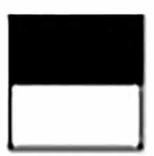

(2)

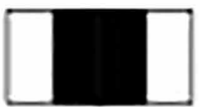

(6)

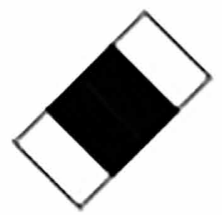

(10)

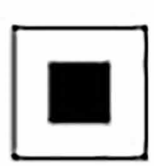

(13)
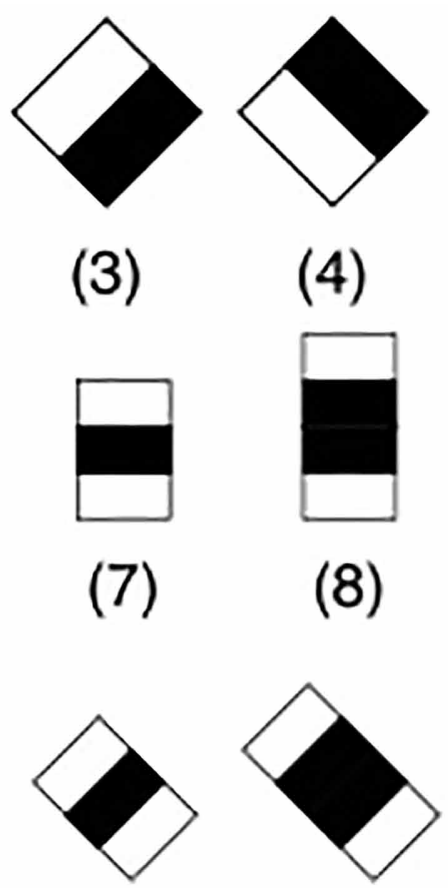

(3)

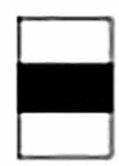

(7)

(11)

(12)

(4)

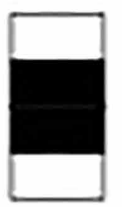

(8)

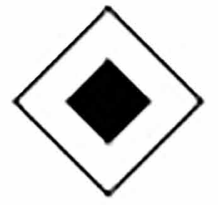

(14)

The classifier $\mathrm{f}$ is an application which is given by Equation (2):

$f: \mathbb{R}^{d} \rightarrow[0,1]^{m}$

$f\left(x_{j}\right)=\left(\mu_{1}\left(V_{x j}\right) ; \ldots ; \mu_{m}\left(V_{x j}\right)\right)$

where $\mu_{i}\left(x_{j}\right)$ represents the degree of membership of the eyes $x_{j}$ to the class $C_{i}$ Each class is represented by a characteristic vector $\mathrm{V}_{\mathrm{ci}}$ :

$V_{C i}=\left(P_{i 1}^{\prime} ; P_{i 2}^{\prime} ; \ldots ; P_{i d}^{\prime}\right)$ 
where:

$\mathrm{P}_{\mathrm{ij}}^{\prime}=\frac{\sum_{\mathrm{k}=1}^{\mathrm{N}} \mathrm{P}_{\mathrm{ij}}[\mathrm{k}]}{\mathrm{N}}$ for $1 \leq \mathrm{i} \leq \mathrm{m}$, where $\mathrm{N}$ is the number of images in the class

$P_{i j}[k]$ represents the characteristic point of number $\mathrm{j}$ found with the Harris method (Harris \& Stephens, 1988) in the image of number $\mathrm{k}$ in the class

The degree of membership is determined by calculating the vector of distances $\mathrm{V}_{\mathrm{d}}$ :

$\mathrm{V}_{d}=\left(\mathrm{D}_{\mathrm{C}_{1}}(\mathrm{x}), \mathrm{D}_{\mathrm{C}_{2}}(\mathrm{x}), \ldots, \mathrm{D}_{\mathrm{C}_{\mathrm{m}}}(\mathrm{x})\right)$

where $\mathrm{D}_{\mathrm{C}_{\mathrm{i}}}(\mathrm{x})$ is the Euclidean distance between $V_{C i}$ and $V_{x j}$ and $\mathrm{m}$ the number of classes.

This fuzzy classification will produce a vector $\mathrm{M}^{\prime}$ :

$$
\mathrm{M}^{\prime}=\left(\mu_{1}\left(V_{x j}\right) ; \ldots ; \mu_{m}\left(V_{x j}\right)\right)
$$

where:

$\mu_{i}\left(V_{x j}\right)=\frac{\mathrm{D}_{\mathrm{C}_{\mathrm{i}}}(\mathrm{x})}{\sum_{\mathrm{j}=1}^{\mathrm{m}} \mathrm{D}_{\mathrm{C}_{\mathrm{j}}}(\mathrm{x})}$ and $\sum_{\mathrm{i}=0}^{\mathrm{m}} \mu_{i}\left(V_{x j}\right)=1$

\section{Fuzzy System of Detection}

\section{Fuzzy Controller}

The different forms of the eyes as well as the methods of image acquisition introduce an imprecision on the eyes detection. This inaccuracy is due to spatial sampling. Through the application of the fuzzy logic in the image processing, we can develop a set of approaches in order to develop several powerful software systems (Reshmalakshmi \& Sasikumar, 2017; Soltani et al., 2018). Fuzzy logic is used in image processing to represent semantic knowledge about image content or processing and decision strategies. On the other hand, the fuzzy sets allow to represent the approach, the contours, the regions, the classes or other structures in the images (Bloch \& Maitre, 1995).

The first step consists of determining the input and output of the linguistic variables. A linguistic variable corresponds to the triplet $(\mathrm{V}, \mathrm{X}, \mathrm{TV})$. $\mathrm{V}$ is the variable, $\mathrm{X}$ is the range of the variable's values and TV is a finite or infinite set of fuzzy subsets. In this paper, the linguistic variables used are the distance between the input eyes and the gravity's center of each class of the database, as well as the points number of interest in the treated eyes that has a correspondence in the images of the database.

\section{Fuzzification}

Fuzzification consists of finding the degree of membership to the linguistic terms describing the calculated attribute. The feature image is delivered at the fuzzification step which translates the values into degrees of membership to the linguistic terms representing the resemblance between the 
detected eyes and the set of eyes recorded in the database. In this phase, the quality of the image is an essential factor to have better results. For example, unclear images may give an incorrect characteristic vector. To remedy this problem, we proposed two methods for this modification, one based on expert judgment and the other is an automatic (Chow \& Li, 1993).

The Euclidian distances are calculated between the detected eyes and the various classes in the database. Then, at each calculated distance, a linguistic value (low similarity, average similarity, high similarity) is matched with a degree of belonging.

Let the fuzzy subsets have low similarity (LS), average similarity (AS) and high similarity (HS) as show in Figure 5.

Figure 5. The distance variable and fuzzy sets used (low similarity (LS), average similarity (AS), high similarity (HS))

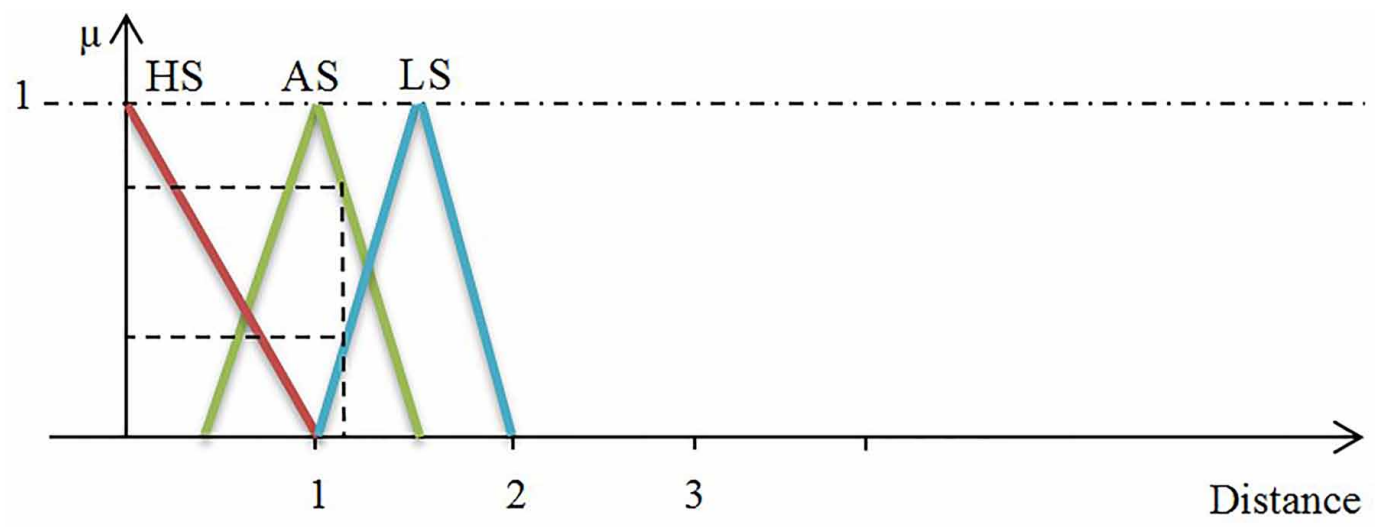

When the distance between the characteristic vector of the input eyes and the gravity's center of the class is sufficiently small, the similarity becomes strong. Therefore, the membership degree for the high similarity subset is big whereas the opposite for the average and low similarity subsets.

To increase the quality of the results, the points of interest's number found in the input image which has a correspondence in the images of the class is a variable that influences the state of the similarity.

Figure 6 shows the experimental results of membership degrees related to the variable number of characteristic points and fuzzy subsets.

From the experimental results (Figure 6), when the number of characteristic points is less than four, the input image does not correspond to any class in the database. In other words, the belonging degree of the variable to the fuzzy subset is zero. But when the number of points becomes greater than or equal to five, a low similarity is noticed. Consequently, the number of points is very important to find the membership's percentage of the image to each class. In addition, when this number exceeds twelve, the classification becomes very close to reality.

\section{Inference System}

After the fuzzification step, it is necessary to define rules that explain how the similarity between the input image and the classes of the database behave as a function of the distance and the number of characteristic points found. The inference system is used to represent the relationship between the input and output variables of the system (Bouchon-Meunier et al., 2005). Several approaches are used to generate fuzzy inference systems (Bandemer \& S. Gottwald, 1995). The general inference system architecture is based on fuzzy rules. A fuzzy rule is a combination of input variables. A combination uses fuzzy operators, such as "AND" and "OR". 
Figure 6. The number of points variable and fuzzy sets used (low similarity (LS), average similarity (AS), high similarity (HS))

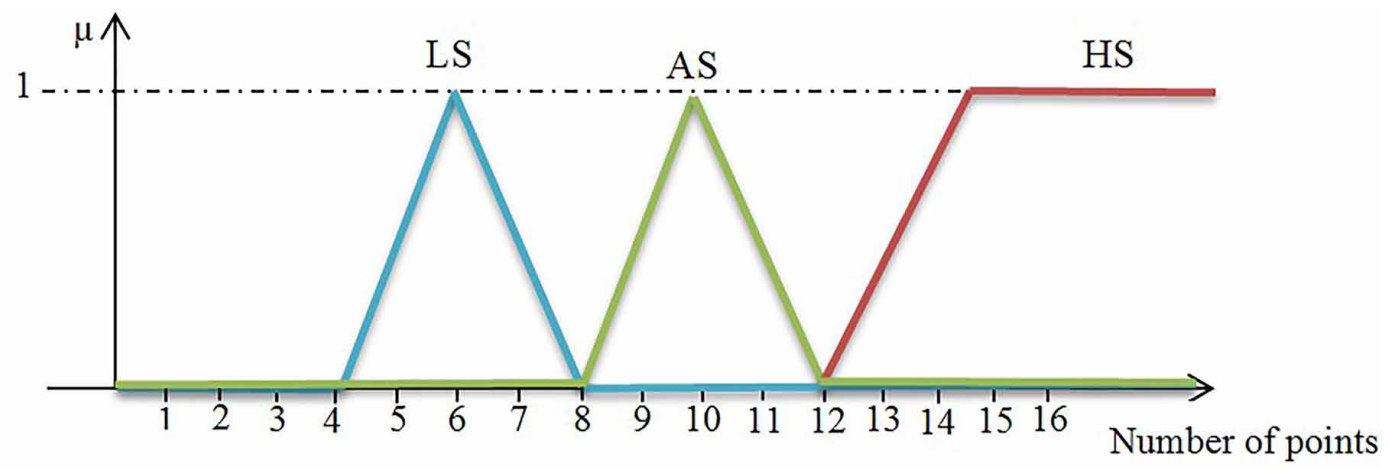

The models of Mamdani (Asadi, 2016) allow to make a linguistic description of the system by a set of fuzzy rules of the form (Alcalá et al., 1999).

If $\left(\mathrm{x}_{1}\right.$ is $\mathrm{A}_{1}$ and $\mathrm{x}_{2}$ is $\mathrm{A}_{2}$ and $\ldots$ and $\mathrm{x}_{\mathrm{n}}$ is $\left.\mathrm{A}_{\mathrm{n}}\right)$ then

$\mathrm{y}_{1}$ is $\mathrm{B}_{1}$ and $\mathrm{y}_{2}$ is $\mathrm{B}_{2}$ and $\ldots$ and $\mathrm{y}_{\mathrm{m}}$ is $\mathrm{B}_{\mathrm{m}}$

where $\mathrm{x}_{\mathrm{i}}$ and $\mathrm{y}_{\mathrm{j}}$ are respectively the language input and output variables and $\mathrm{A}_{\mathrm{i}}$ and $\mathrm{B}_{\mathrm{j}}$ are the fuzzy linguistic sets.

Figure 5 and Figure 6 shows the relationships used to generate the set of inference rules. The distance between the eyes of the persons treated and the classes of the database makes, and the number of characteristic points it possible to give the percentage of membership of each fuzzy set, which makes it possible to take a decision. Several rules can be used in the inference database. For example:

If (distance is HS and Number of points is HS) then

Similarity is HS

\section{Data Base}

The database consists of an M matrix that contains interest's points of all images (Figure 7) taken by a canon MARK II 5D camera as well as images from the ferret and ESSEX face database libraries. Then, a median filter is applied to the images taken by the camera. The construction of the vectors characteristic of the input images is done by the method of Harris (Harris \& Stephens, 1988) (Figure 8). This method allows returning a set of the interest's points $\left(\mathrm{P}_{\mathrm{ij}}\right)$ where $\mathrm{i}$ is the class number and $\mathrm{j}$ is the sequence number of the point (Figure 7).

\section{Defuzzification}

Defuzzification consists of applying the chosen fuzzy rule. In this step, our objective is to keep only the classes $\mathrm{C}_{\mathrm{i}}$ whose membership's degree of the eye $\mathrm{x}_{\mathrm{j}}$ is greater than or equal to a given threshold, the detected eyes can now belong to several classes. 


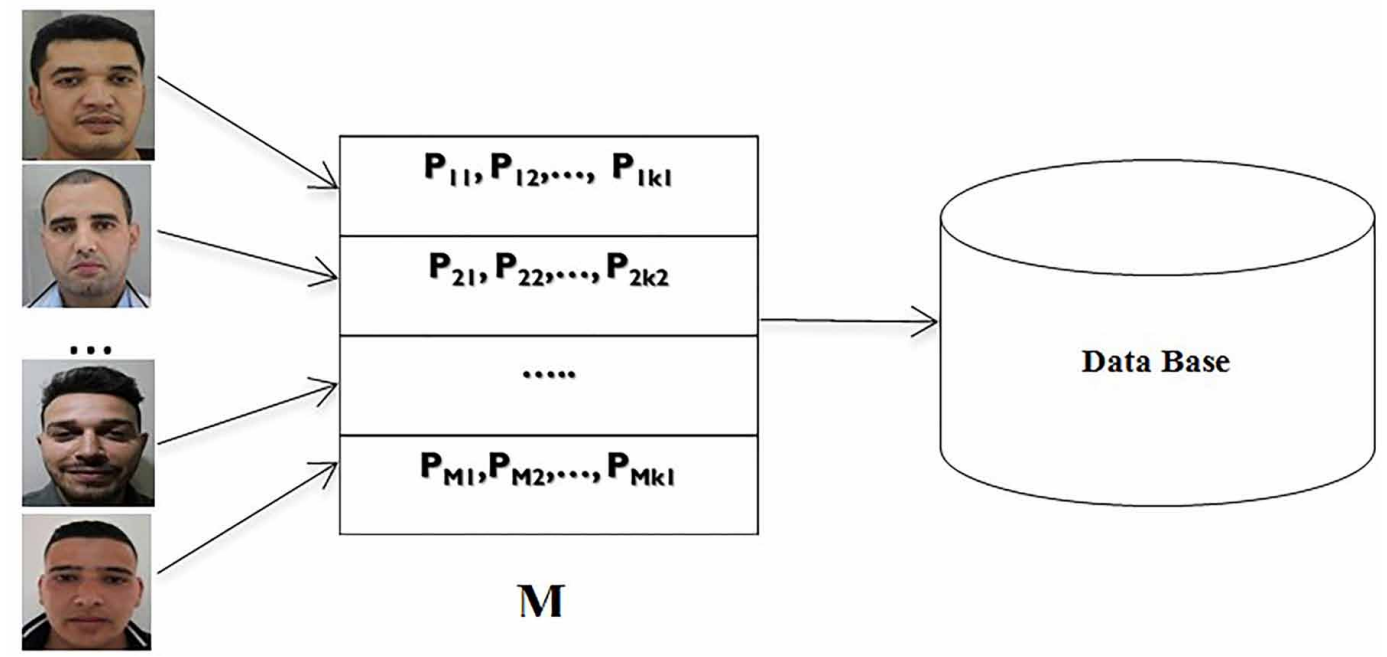

Figure 8. Extraction of points of interest with Harris corner method

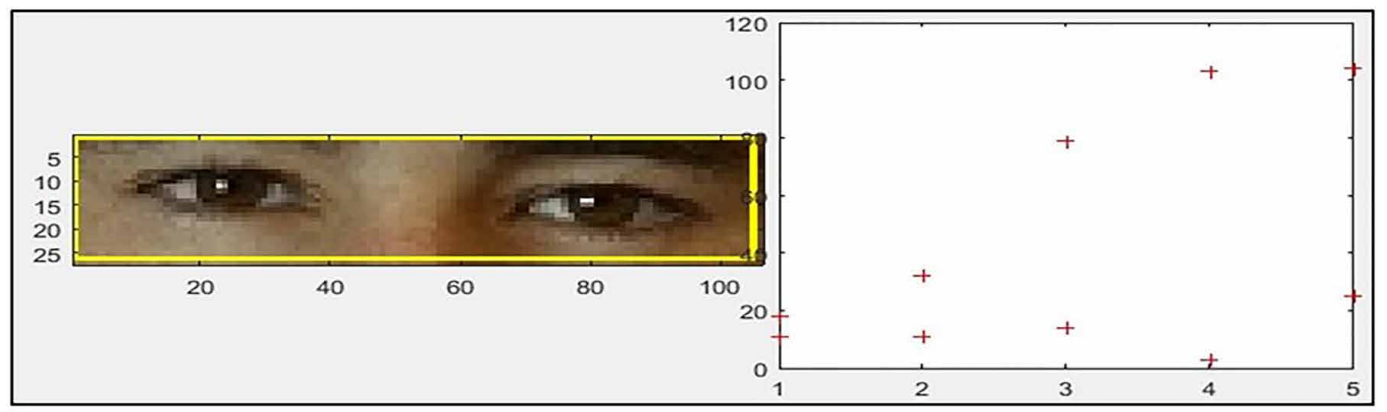

\section{RESULTS AND DISCUSSION}

We conducted several tests to prove the reliability of our study. For each image, we have made a comparison between the algorithm used in this paper and the other algorithms that exist in the domain (Table 1). Our database of images contains 200 images; we took a sample of 30 images to do the tests.

The different groups participating in the test are from different origins. Group one contains image with black skin and group two consist people who has a white skin; whereas group three contains image who use glasses (Figure 9).

Method 1 used in the tests represents the method of D. Ram Nivas, T. Manigandan (Nivas \& Manigandan, 2015) and method 2 represents the method of Mark Everingham and Andrew Zisserman (Everingham \& Zisserman, 2006). Each classification method has advantages and disadvantages, and the choice of the right method depends on the intended application. The first method (Nivas \& Manigandan, 2015) is based on the calculation of the total number of key points extracted and the pupil diameter. The second method (Everingham \& Zisserman, 2006) uses a Bayesian approach for eye classification. 
Table 1. Results of classification obtained by three methods

\begin{tabular}{|l|l|l|l|l|l|l|}
\hline & \multicolumn{4}{l}{ Detection methods } & \multicolumn{2}{l|}{ Our Method } \\
\hline Pictures & Method 1 & \multicolumn{2}{l|}{ Method 2 } & $3.15 \mathrm{~s}$ & $86 \%$ & $3.34 \mathrm{~s}$ \\
\hline Group 1 & $85 \%$ & $3.5 \mathrm{~s}$ & $74.9 \%$ & $4.2 \mathrm{~s}$ & $78 \%$ & $4 \mathrm{~s}$ \\
\hline Group 2 & $73 \%$ & $4 \mathrm{~s}$ & $41 \%$ & $2.4 \mathrm{~s}$ & $83 \%$ & $2.3 \mathrm{~s}$ \\
\hline Group 3 & $81 \%$ & $2.2 \mathrm{~s}$ & $77 \%$ & & \\
\hline
\end{tabular}

Figure 9. Examples of the images used to do the tests

Group 1

Group 2

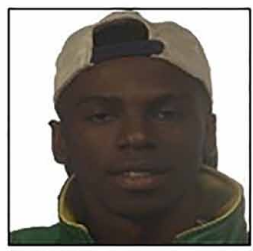

Group 3
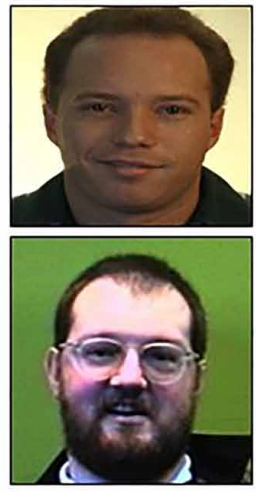
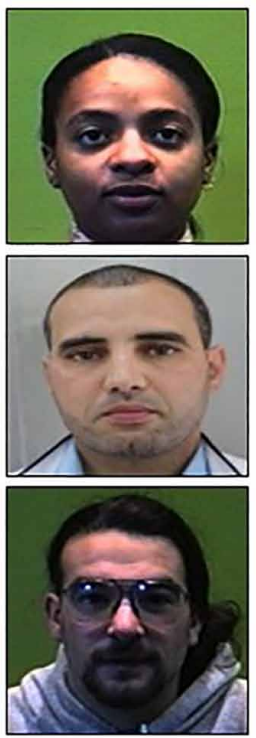
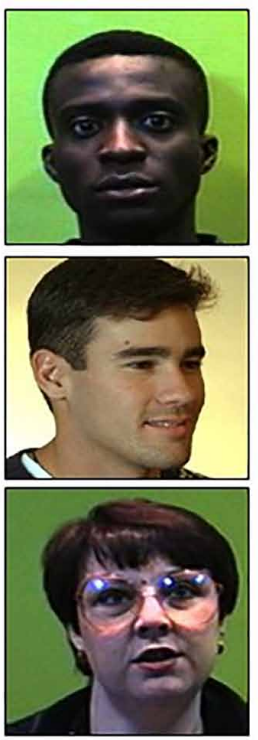
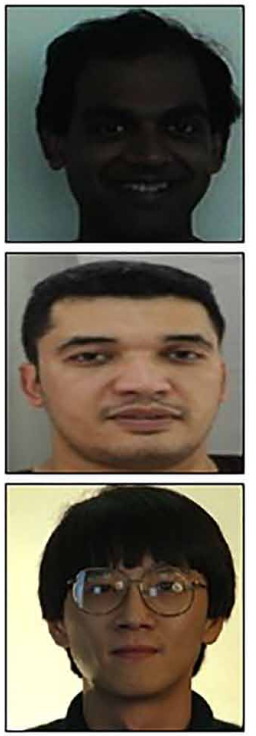

\section{CONCLUSION}

In this paper, a detection method based on fuzzy logic approach is proposed. A prototype of an eye classification system is based on fuzzy logic through several steps. The first step is to detect the person's eyes, then, the result of the detection is followed by a fuzzification phase. After that, a set of inference rules is applied, and a defuzzification phase is done in the final step. This system used for eye classification is robust and should help researchers in several fields. The results were generally satisfactory.

From the results obtained, fuzzy logic has made it possible to achieve a powerful classification system; however, other improvements in the quality of acquired images may be added to speed up processing. Among the problems encountered with this method is the classification when the eyes are partially visible. 


\section{REFERENCES}

Alcalá, R., Casillas, J., Cordón, O., \& Herrera, F. (1999). Approximate Mamdani-type Fuzzy Rule-Based Systems: Features and Taxonomy of Learning Methods.

Asadi, M. (2016). Optimized Mamdani fuzzy models for predicting the strength of intact rocks and anisotropic rock masses. Journal of Rock Mechanics and Geotechnical Engineering, 8(2), 218-224. doi:10.1016/j. jrmge.2015.11.005

Bandemer, H., \& Gottwald, S. (1995). Fuzzy Sets, Fuzzy Logic, Fuzzy Methods with Applications. Chichester: J. Wiley.

Bloch, I. (2003). Fusion d'informations en traitement du signal et des images. Hermes Science Publication.

Bloch, I., \& Maitre, H. (1995). Fuzzy mathematical morphologies: A comparative study. Pattern Recognition, 28(9), 1341-1387. doi:10.1016/0031-3203(94)00312-A

Bouchon-Meunier, B., Esteva, F., Godo, L., Rifqi, M., \& Sandri, S. (2005). A principled approach to fuzzy rule base interpolation using similarity relations. In Proceedings of the EUSFLAT--LFA 2005 (pp. 757-763). Academic Press.

Chow, G., \& Li, X. (1993). Toward a system for automatic facial feature detection. Pattern Recognition, 26(12), 1739-1755. doi:10.1016/0031-3203(93)90173-T

Christensen, J. A. E., Kempfner, L., Leonthin, H. L., Hvidtfelt, M., Nikolic, M., Kornum, B. R., \& Jennum, P. (2017). Novel method for evaluation of eye movements in patients with narcolepsy. Sleep Medicine, 33, 171-180. doi:10.1016/j.sleep.2016.10.016 PMID:28087252

Cleveland, N. (1994). Eyegaze Human-computer interface for people with disabilities. In Proceedings of the First Automation Technology and Human Performance Conference. Academic Press.

Dimililer, K., Ever, Y. K., \& Ratemi, H. (2016). Intelligent eye Tumour Detection System. Procedia Computer Science, 102, 325-332. doi:10.1016/j.procs.2016.09.408

Everingham, M., \& Zisserman, A. (2006). Regression and Classification Approaches to Eye Localization in Face Images. In Proceedings of the 7th International Conference on Automatic Face and Gesture Recognition (FGR06) (pp. 441-448). IEEE Press. doi:10.1109/FGR.2006.90

Harris, C., \& Stephens, M. (1988). A combined corner and edge detector. In Proc. of Fourth Alvey Vision Conference (pp. 147-151). Academic Press.

Heuvelink, G. B. M., Schoorl, J. M., Veldkamp, A., \& Pennock, D. J. (2006). Space-time Kalman filtering of soil redistribution. Geoderma, 133(1), 124-137. doi:10.1016/j.geoderma.2006.03.041

Hoya, T., Hori, G., Bakardjian, H., Nishimura, T., Suzuki, T., \& Miya-waki, Y., ... J. Cao. (2003). Classification of Single Trial EEG Signals by a Combined Principal + Independent Component Analysis and Probabilistic Neural Network Approach. In Proceedings ICA2003 (pp. 197-202). Academic Press.

Liu, S., Niu, Z., Sun, G., \& Chen, Z. (2014). Gabor filter-based edge detection : A note. Optik (Stuttgart), 125(15), 4120-4123. doi:10.1016/j.ijleo.2014.01.102

Lukac, R. (2003). Adaptive vector median filtering. Pattern Recognition Letters, 24(12), 1889-1899. doi:10.1016/ S0167-8655(03)00016-3

Minhad, K. N., Ali, S. H. M., \& Reaz, M. B. I. (2017). Happy-anger emotions classifications from electrocardiogram signal for automobile driving safety and awareness. Journal of Transport \& Health, 7, 75-89. doi:10.1016/j.jth.2017.11.001

Nivas, D. R., \& Manigandan, T. (2015). Classification of Human Eye Image using different Methods for Medical Applications. International Journal of Advanced Research in Computer and Communication Engineering, 4(4).

Reshmalakshmi, C., \& Sasikumar, M. (2017). Image Edge Orientation Estimation via Fuzzy Logic. Materials Today: Proceedings, 4(2, Part B), 4274-4282. 
Rigas, I., \& Komogortsev, O. V. (2017). Current research in eye movement biometrics: An analysis based on BioEye 2015 competition. Image and Vision Computing, 58, 129-141. doi:10.1016/j.imavis.2016.03.014

Soltani, A., Battikh, T., Jabri, I., \& Lakhoua, N. (2018). A new expert system based on fuzzy logic and image processing algorithms for early glaucoma diagnosis. Biomedical Signal Processing and Control, 40, 366-377. doi:10.1016/j.bspc.2017.10.009

Song, K., Shen, F., Liu, Z., \& Liu, Z. (2010). Eye Detection and Recognition in the Fatigue Warning System. In Proceedings of the 2010 Third International Conference on Intelligent Networks and Intelligent Systems (pp. 36-38). Academic Press. doi:10.1109/ICINIS.2010.22

Uchino, M., Kawashima, M., Uchino, Y., Suzuki, N., Mitamura, H., Mizuno, M., \& Tsubota, K. et al. (2018). The evaluation of dry eye mobile apps for screening of dry eye disease and educational tear event in Japan. The Ocular Surface, 16(4), 430-435. doi:10.1016/j.jtos.2018.06.002 PMID:29883737

Viola, P., \& Jones, M. (2001). Rapid object detection using a boosted cascade of simple features. In Proceedings of the 2001 IEEE Computer Society Conference on Computer Vision and Pattern Recognition. CVPR 2001 (pp. I-511-I-518). IEEE. doi:10.1109/CVPR.2001.990517

Yuille, A. L., Cohen, D. S., \& Hallinan, P. W. (1989). Feature extraction from faces using deformable templates. In Proceedings CVPR '89: IEEE Computer Society Conference on Computer Vision and Pattern Recognition (pp. 104-109). IEEE Press. doi:10.1109/CVPR.1989.37836

Mohamed Fakir is a professor at Sultan Moulay Slimane university. He obtained a master's degree from Nagaoka university of technology and PHD from Cadi Ayyad university. His research interest includes pattern recognition, datamining and artificial intelligence.

Hicham Hatimi is PHD student. His research interest includes pattern recognition, image processing and datamining Mohamed Chabi is a professor at Sultan Moulay Slimane university.

Muhammad Sarfraz is a Professor and Director of MSIT in the Department of Information Science, Kuwait University, Kuwait. His research interests include computer graphics, pattern recognition, computer vision, image processing, soft computing, data science, intelligent systems, information technology, and information systems. He is currently working on various projects related to academia and industry. Prof. Sarfraz has been keynote/invited speaker at various platforms around the globe. He has advised/supervised more than 85 students for their MSc and PhD theses. He has published more than 350 publications as journal and conference papers. His publications include around 60 Books as Autor and Editor. He is also editor of proceedings books of various conferences around the globe. Prof. Sarfraz is member of various professional societies including IEEE, ACM, IFAC, IVS, INSTICC and ISOSS. He is a Chair and member of the International Advisory Committees and Organizing Committees of various international conferences, Symposiums and Workshops. He is also editor-in-chief, editor and guest editor of various international journals. He is the reviewer, for many international journals, conferences, meetings, and workshops around the world. He has achieved various awards in education, research, and administrative services. 